vol. $27-n^{\circ} 2$ | 2011

Numéro ouvert

\title{
Consommation et cultures de la différence chez les jeunes descendants d'immigrés. Réflexions à partir du cas italien
}

Consumption and Cultures of Difference among the Children of Immigrants.

Reflections from the Italian Case

Comsumos y culturas de la diferencia de los hijos de inmigrantes. Reflexión

sobra lo caso Italiano

\section{Paola Rebughini}

\section{(2) OpenEdition}

\section{Journals}

Édition électronique

URL : https://journals.openedition.org/remi/5441

DOI : 10.4000/remi.5441

ISSN : $1777-5418$

Éditeur

Université de Poitiers

Édition imprimée

Date de publication : 1 octobre 2011

Pagination : 101-116

ISBN : 979-10-90426-01-6

ISSN : 0765-0752

Référence électronique

Paola Rebughini, «Consommation et cultures de la différence chez les jeunes descendants

d'immigrés. Réflexions à partir du cas italien », Revue européenne des migrations internationales [En

ligne], vol. $27-n^{\circ} 2$ | 2011, mis en ligne le 01 octobre 2014, consulté le 15 avril 2022. URL : http://

journals.openedition.org/remi/5441; DOI : https://doi.org/10.4000/remi.5441 


\section{Consommation et cultures de la différence chez les jeunes descendants d'immigrés. Réflexions à partir du cas italien}

\section{Paola REBUGHINI ${ }^{1}$}

es études sur la consommation sont souvent considérées comme un secteur
spécifique de la sociologie qui entre rarement en contact avec celui des recherches sur les migrations. C'est seulement dans des études plus récentes, par exemple celles sur la circulation des individus et des biens dans les espaces marchands transnationaux et déterritorialisés (Battegay, 2003 ; Tarrius, 2002 ; Raulin, 2000), que cette rencontre a pu montrer toutes ses potentialités.

Cet article se concentre sur des acteurs spécifiques - les descendants d'immigrés en Italie - et sur leur rapport à la consommation et à la culture matérielle, dans une tentative de comprendre l'articulation entre les modèles d'identification et d'appartenance de cette génération de jeunes et leurs pratiques quotidiennes de relations aux objets. Pour essayer de comprendre cette articulation, je vais m'appuyer sur les résultats de cinq années de recherche en équipe (2004-2009) sur les enfants d'immigrés dans la région milanaise et en particulier sur une recherche spécifiquement dédiée aux pratiques et aux cultures de la consommation chez cette catégorie de jeunes (Rebughini, 2010)2.

Les modèles et les cultures de la consommation sont apparus comme une perspective particulièrement intéressante pour observer à la fois les aspects structuraux de l'intégration, liés à la position socioéconomique des familles immigrées, et les aspects interprétatifs et symboliques de la différence culturelle qui caractérisent les pratiques quotidiennes des enfants avec des parents étrangers. En effet, le point de vue de la consom-

1 Maître de conférence, chercheur, Département d'études sociales et politiques, Université de Milan, Via Conservatorio 7, 20122 Milano, Italie ; paola.rebughini@unimi.it

2 Depuis 2004 nous avons réalisé une série de recherches qualitatives - qui ont concerné environ 250 jeunes entre quatorze et vingt et un ans de différentes nationalités ou origines nationales dédiées à l'étude des modèles d'identifications, des sentiments d'appartenance et aux pratiques de la vie quotidienne caractéristiques de cette génération de descendants d'immigrés (Bosisio, Colombo, Leonini, Rebughini, 2005 ; Colombo, Leonini, Rebughini, 2009). La recherche spécifiquement dédiée aux modèles de consommation a concerné soixante-treize jeunes. 
mation présente l'intérêt d'associer les aspects contingents et subjectifs des pratiques de consommation avec les aspects qui relèvent au contraire des ressources économiques et culturelles plus stables dans le temps (Zukin et Smith-Maguire, 2004 ; Langlois, 2002)3.

Après une rapide présentation du contexte italien et de la situation générale des jeunes descendants d'immigrés dans ce pays, je vais présenter les principaux résultats de la partie de recherche dédiée aux cultures et pratiques de consommation chez les enfants d'immigrés à Milan.

\section{LE CONTEXTE ITALIEN : OPPORTUNITÉS ET LIMITES D'UNE GÉNÉRATION À L'ÉPREUVE}

Dans l'Italie contemporaine, la présence des jeunes descendants d'immigrés dans l'espace public et surtout dans les écoles est considérée comme une donnée acquise. Il s'agit cependant d'un changement très récent qui s'est développé dans les dix dernières années et dont il faut encore comprendre dans le détail les caractéristiques. Les études quantitatives aujourd'hui disponibles nous montrent pour l'instant une situation d'intégration progressive et sans secousses sociales importantes - exception faite bien évidemment dans le domaine scolaire - pour cette génération, presque un million de mineurs dont la moitié est née en Italie (Caritas Migrantes, 2009 ; Della Zuanna, Farina et Strozza, 2009). Les études qualitatives, à travers des instruments d'observation plus fins et localisés, nous montrent de leur côté les nuances des trajectoires biographiques et scolaires de ces jeunes, leurs tactiques d'identification, les approches à la citoyenneté et à l'appartenance ou encore la construction de liens sociaux caractérisés par l'ambivalence et la flexibilité plutôt que par la cohérence et l'exclusivité (Ambrosini et Molina, 2004 ; Ambrosini et Queirolo Palmas, 2005 ; Valtolina et Marazzi, 2006 ; Queirolo Palmas, 2006 ; Colombo, Domaneschi et Marchetti, 2009 ; Colombo, Leonini et Rebughini, 2009 ; Besozzi, Colombo et Santagati, 2009 ; Rebughini et Leonini, 2010).

Il existe en effet une spécificité historique et générationnelle de l'expérience du passage à l'âge adulte chez les jeunes descendants d'immigrés en Italie : il s'agit d'une génération qui a grandi dans un contexte historique postindustriel et technologique qui favorise le contact et la familiarité avec la différence culturelle, la circulation des flux d'informations et des images, ainsi que des formes de multiculturalisme quotidien. Le contexte italien actuel se caractérise par une grande hétérogénéité des flux migratoires et par la présence très limitée de concentrations urbaines de type communautaire, ce qui favorise le pluralisme des descendances nationales des enfants d'immigrés dans les écoles et dans les quartiers. Ce milieu potentiellement multiculturel semble aussi favorisé par l'absence d'une symbiose explicite entre culture nationale et valeurs universelles et par l'absence de politiques publiques orientées à renforcer la différence culturelle et la communauté ethnique, comme cela s'est vérifié aux États-Unis et dans d'autres pays

3 Contrairement aux premières études sur la consommation - caractérisées par une interprétation en termes de domination et de conditionnement incontournable du consommateur - les études contemporaines ont plutôt tendance à souligner la souplesse adaptative de la culture matérielle et la pluralité des significations caractéristiques des choix d'achat dans toutes les classes d'âge et dans différents milieux culturels (Miller, 1995 ; Warde, 2005). 
européens plus précocement investis par les flux migratoires. La généalogie de la position sociale « d'enfant d'immigrés » est également différente par rapport aux pays avec un passé colonial important et une identité nationale historiquement consolidée.

Par ailleurs, l'Italie, état-nation qui remonte seulement au XIXe siècle, est toujours un pays culturellement très hétérogène avec des différences régionales importantes. Ce dernier point entraîne une grande diversité des expériences locales d'intégration des immigrés et de leurs enfants et s'associe à une certaine flexibilité institutionnelle. En effet, si les politiques sociales nationales, malgré leurs insuffisances, ont favorisé une intégration plus générale des migrants et de leurs descendants, les politiques sociales régionales et municipales restent très diversifiées.

Tout cela semble avoir favorisé chez ces jeunes une certaine autonomie subjective dans le rapport à ses propres origines familiales et avoir facilité la construction de liens sociaux capables d'inclure et de mobiliser des ressources identitaires différentes, plutôt que de les subir ou de les simplifier dans une identification exclusive.

Évidemment cela ne signifie pas qu'il n'y ait pas de problèmes qui guettent le futur de cette génération : être capable de gérer plusieurs identifications sans s'enfermer dans une différence culturelle, ou au contraire dans des formes de mimétisme, demande des ressources et des capacités personnelles qui relèvent aussi de l'éducation familiale et du milieu social. Le capital culturel et social de la famille, notamment, est central dans les choix scolaires, mais aussi dans la capacité de transmettre à ses propres enfants une certaine ouverture d'esprit nécessaire à gérer d'une façon autonome les codes et les symboles de différentes cultures. En effet, cela est indispensable pour savoir utiliser sa propre différence comme une ressource de distinction plutôt que comme le signe d'un stigmate subi. Or, les enquêtes statistiques nous montrent une concentration de plus en plus évidente des fils d'immigrés dans les lycées professionnels et en général dans les filières scolaires les moins qualifiées, ainsi qu'une concentration de leurs familles dans les couches sociales les moins favorisées. Cela semble suivre une tendance déjà présente dans d'autres pays (Payet, 2008 ; Portes, Ferandez-Kelly et Haller, 2005 ; Roulleau-Berger, 2009) et révéler le risque d'une mobilité sociale bloquée, ou de formes d'intégration vers le bas, selon le processus décrit dans la littérature américaine comme downward assimilation (Portes et Rumbaut, 2001).

Un deuxième point de tension est ensuite représenté par la loi italienne sur la citoyenneté, centrée sur le principe du « droit du sang » (jus sanguinis) qui pénalise les jeunes descendants d'immigrés et rend difficiles et extrêmement pénibles les démarches bureaucratiques pour leur naturalisation. On estime à environ un million, le nombre de jeunes adultes d'origine étrangère qui attendent la citoyenneté italienne et des recherches récentes montrent toute la frustration de ces jeunes par rapport à l'impossibilité d'avoir accès à la nationalité, malgré leur sentiment d'être « italiens comme les autres » (Colombo, Domaneschi et Marchetti, 2009).

Pour résumer, il est possible d'affirmer qu'aujourd'hui les adolescents fils d'immigrés en Italie profitent d'un avantage important par rapport aux précédentes générations d'enfants d'immigrés en Europe : celui de vivre dans un contexte historique plus cosmo- 
polite et plus familiarisé à la différence culturelle. Cependant ils doivent faire face aussi à un certain nombre de défis. Ces jeunes sont en effet pénalisés par la nécessité de gérer des épreuves qui caractérisent l'ensemble de leur génération : montée du chômage, précarité du travail, mobilité sociale bloquée, risque d'une intégration subalterne, présence de plus en plus visible d'un discours discriminatoire dans les médias et dans l'espace public. Chacun de ces jeunes est appelé personnellement à répondre à ces défis, car la protection que les communautés ou les réseaux ethniques peuvent offrir - par ailleurs plutôt faiblement développés en Italie - ne compense pas l'individualisation de ces épreuves (Martuccelli, 2006).

\section{LA CONSOMMATION COMME FORME D'INTÉGRATION ?}

Le concept d'intégration met parfois mal à l'aise le sociologue, car il peut évoquer une idée fonctionnaliste de la socialisation et de l'inclusion dans une culture majoritaire qui se conçoit comme exclusive et universelle (Wieviorka, 2008). Un usage plus faible et souple du concept d'intégration peut cependant se lier à une idée de participation à la société dans laquelle les jeunes descendants d'immigrés vivent, sans s'associer à une idée exclusive d'appartenance. Dans ce sens, la consommation peut être envisagée comme un moyen de participation parmi d'autres, sans la considérer nécessairement comme une forme d'acculturation à un modèle majoritaire euro-américain ou consumériste. Certes, il existe des différences parmi les jeunes consommateurs. Comme on va le voir, certains jeunes d'origine étrangère sont capables d'innover dans leurs pratiques de consommation et d'attribuer des significations nouvelles et personnelles aux objets de consommation quotidienne, à travers l'utilisation de leur différence culturelle comme ressource. À l'opposé, il y a aussi des jeunes qui considèrent un style de consommation mimétique et conforme à celui de la majorité comme la première et indispensable source d'inclusion sociale.

Considérer que les adolescents - toutes origines confondues - sont des consommateurs passifs et facilement manipulables est une représentation triviale et partiellement démentie par la plupart des recherches sur la consommation, qui montrent plutôt une certaine autonomie du consommateur, y compris pendant l'adolescence. Cela n'empêche que les styles de consommation aient un rôle important dans les stratégies d'intégration sociale, surtout dans les situations où cette intégration semble incertaine ou remise en question. En particulier, dans la littérature française sur le style de vie des jeunes des banlieues (Lapeyronnie, 1998 ; 2009) ou dans la littérature américaine sur la downward assimilation (Portes, Fernandez-Kelly et Haller, 2009), l'injonction à l'intégration semble passer par des modèles de consommation hyper-socialisés qui se manifestent par l'aspiration à surmonter l'exclusion sociale par l'accès à certaines marques et objets. Dans ces portraits, la vie quotidienne des jeunes se caractérise donc par la contradiction entre un modèle plus ou moins construit d'intégration consumériste et des formes explicites et concrètes de ségrégation urbaine et sociale.

Dans le cadre que nous avons observé chez les jeunes descendants d'immigrés à Milan, cependant, les comportements de consommation marchande conformistes et mimétiques ne semblent pas le produit d'une injonction à une forme contraignante d'in- 
tégration sociale, ni la réaction passive d'une population qui se perçoit comme exclue. Il s'agit plutôt d'un désir d'intégration et d'identification plus souple, qui passe d'abord par le groupe des jeunes du même âge, donc par le désir d'avoir les mêmes objets de référence.

" J'aime bien m'habiller selon la mode et avoir le même style que mes copines, par exemple les pantalons bas : tout le monde les porte, alors pourquoi pas moi? Je les porte aussi... ma grand-mère, au contraire, les trouve moches et peu élégants, mais je lui réponds que c'est à la mode, que je veux m'habiller comme mes copines et que, quand la mode sera passée, j'utiliserai d'autres pantalons » (C. 16 ans (F) parents cubains).

"J'aime bien le shopping : le samedi, je vais toujours au centre-ville avec mes amis pour acheter quelque chose, mon père me donne vingt euros par semaine, mais

si j'ai besoin de plus, il me donne plus d'argent, ou bien je garde les vingt euros pour plusieurs semaines, car les chaussures et les vêtements de marque sont chers... J'aime être habillé comme il faut, être en ordre, j'aime les belles choses » (A. 18 ans

(M) parents marocains).

« Je suis habillé comme les autres, normal quoi! Certes je n'achète pas les chaussures chez les Chinois du marché, car je vais chez Footlocker avec mes amis.

Quand je dois acheter quelque chose de marque au centre-ville je vais avec mes amis, pas avec ma mère... ma mère achète pour mois des T-shirt au marché, les T-shirt blancs tu sais, pour la maison ou pour la nuit... » (M. 17 ans (M) parents péruviens).

Chez les adolescents, la priorité est d'être accepté par le groupe, car dans cette classe d'âge les amis constituent une référence fondamentale. Les goûts et les pratiques de consommation sont donc influencés par cette exigence, surtout en ce qui concerne la présentation de soi dans l'espace public, donc envers l'habillement. Au contraire, d'autres types de consommation - musicales ou alimentaires notamment - peuvent être privés et non partagés avec les autres jeunes du même âge, révélant une liberté et une variabilité bien supérieure.

Les attitudes conformistes dans l'habillement sont parfois soutenues par les parents qui, à leur tour, sont convaincus qu'un style de consommation en ligne avec la majorité est un vecteur important pour l'intégration sociale de leurs enfants et l'épanouissement de leur personnalité, ou pour éviter qu'ils soient discriminés. Pour cette raison, même s'il s'agit de familles à revenu modeste, les parents sont parfois prêts à faire face aux exigences de consommation « de marque », y compris via des formes d'emprunt et d'endettement. Cependant, dans ce dernier cas, il s'agit d'une culture familiale des achats qui concerne toute la famille et qui souvent remonte à une socialisation à la consommation reçue par les parents dans le pays d'origine, antérieure à leur installation en Italie. En contrepartie, cette attitude passive et mimétique est stigmatisée par d'autres familles immigrées et par leurs enfants, qui basent au contraire leur projet d'insertion sociale dans la société d'accueil sur un discours de sacrifice et de responsabilité qui refuse toute forme de consumérisme.

Dans tous les cas, les jeunes rencontrés pendant nos recherches semblent capables d'agir à travers une sorte de redéfinition de la culture matérielle qui les entoure. Dans ce sens, bien que les comportements mimétiques, ou le désir d'être habillés avec les 
marques « que tout le monde porte » soient assez répandus, ils entretiennent d'autres types de consommation plus hétérogènes dans le domaine de la musique, de la danse ou des médias, renouant ainsi un lien avec les origines familiales ou avec une culture transnationale qui mélange les styles et les références.

Finalement, bien que leurs pratiques de consommation semblent très semblables à celles de leurs amis italiens de souche, elles demeurent appréhendées à travers une réflexion associée à leur condition de jeunes d'origine étrangère.

"J'aime bien mélanger les accessoires et les vêtements : le voile est toujours associé à la couleur de mon T-shirt ou de ma chemise, porter le voile ne veut pas dire être moche... Je porte les jeans mais avec une chemise suffisamment longue, il faut être suffisamment couverte et sobre dans l'habillement, mais je ne renonce pas à soigner ma personne [...]. Les jeans, les pull-overs, l'anorak, je les achète dans les grands magasins avec ma mère ou mes sœurs, les chemises et les foulards dans des petits magasins que connaît ma mère, ou parfois au marché [...]. Mes copines aiment bien mon habillement : elles le trouvent original, je suis toujours bien rangée, avec des bijoux originaux qu'on ne trouve pas ici, car ils étaient à ma grand-mère. Parfois, $j$ 'achète aussi des bijoux au marché, mais je les porte à ma façon [...]. J'aime bien la musique indienne moderne, très rythmée pour danser, mais j'aime aussi le rock, et je télécharge dans l'ordinateur d'autres morceaux qui me plaisent... » (R. 19 ans (F) parents indiens musulmans).

S'il est vrai - comme les théories les plus récentes de la consommation le soutiennent - que, bien qu'influencées par la position sociale ou les techniques du marketing, les pratiques d'achat sont toujours le résultat singulier et spécifique d'une appréciation personnelle (Warde, 2005), il est possible d'affirmer que le fait d'être né dans une famille immigrée et d'avoir des parents qui appartiennent à des cultures différentes peut constituer une ressource interprétative ultérieure, valorisante et non seulement un handicap et un risque de stigmatisation. Pour cette raison, même l'achat apparemment conformiste de certaines marques-symbole ne peut pas être automatiquement considéré comme une forme d'acculturation et d'assimilation aux pratiques consuméristes occidentales ; mais également - d'une façon moins contraignante - comme une stratégie d'inclusion et une affirmation de la part de jeunes à circuler parmi des modèles d'identifications différents et parmi des esthétiques et pratiques de consommation très différenciées. La culture des parents, même quand elle est critiquée ou négligée, reste une source affective et émotive d'identification : elle influence donc la participation et l'interprétation des cultures de la consommation. C'est la raison pour laquelle, dans les cas des jeunes descendants d'immigrés, les significations personnelles que chaque individu donne à ses actes de consommation s'associent à une élaboration singulière et subjective de leur rapport à la différence transmise par leurs parents, comme cela a été montré aussi par d'autres recherches effectuées dans d'autres pays (Appadurai, 1996 ; Canclini, 1998 ; Chin, 2001 ; Davila, 2001 ; Zukin, 2004).

Par ailleurs, la présence des immigrés et de leurs descendants rend aussi inactuelle la description de la société euro-américaine comme d'un monolithe culturel : le modèle majoritaire de la société de consommation est de moins en moins celui de la « bourgeoisie de l'homme blanc adulte et occidental» (Brewer et Trentmann, 2006), car dans nos sociétés plus transnationales et cosmopolites, les modèles de consommation se 
sont aussi pluralisés et comprennent des formes différentes de consommation, y compris des formes alternatives ou critiques. Ces aspects plus hétéroclites sont bien plus visibles dans la consommation culturelle, musicale et médiatique. Par exemple, interrogés sur ses goûts musicaux, la plupart de ces jeunes révèlent des références tout à fait multiculturelles ; c'est surtout la possibilité de télécharger gratuitement la musique sur Internet qui les pousse à s'approcher de styles différents. Si à la maison, les parents écoutent parfois à la radio la musique du pays d'origine, leurs enfants préfèrent en général l'écouter avec leur ordinateur, ou leur iPod. Cependant, la fracture technologique et générationnelle entre parents et enfants n'est pas nécessairement une fracture culturelle : beaucoup de jeunes écoutent les versions contemporaines des musiques traditionnelles du pays des parents, mais l'associent aussi à l'écoute d'autres styles musicaux.

"Quand je rentre à la maison, j'allume immédiatement mon ordinateur et la télé sur MTV ... J'aime bien écouter les nouveautés dans le monde de la musique pop et rock, mais j'écoute aussi la musique classique, même si je ne supporte pas l'opéra...

j'écoute aussi la musique chinoise moderne. Quand je fais mes devoirs et que mes parents ne sont pas là, j'écoute toujours de la musique, même du hard rock... j'échange aussi beaucoup de morceaux musicaux avec mes amis, ici et en Chine : avec YouTube et le web, c'est facile » (Y. 18 ans (M) parents chinois).

La musique et les médias sont les vecteurs principaux de l'hybridité chez la deuxième génération qui se trouve à l'intérieur d'un soundscape transnational et transversal, dont la nature n'est pas seulement commerciale mais aussi capable de véhiculer une approche omnivore de la culture iconographique et musicale (Appadurai, 1996). En effet, la disponibilité et la facilité d'accès à ces différentes cultures sur le web ont permis une plus grande personnalisation et individualisation dans la consommation d'images et de musique chez cette génération " technologisée », alors que leurs parents sont restés liés à une consommation culturelle radiophonique et télévisuelle, qui propose encore de choisir entre les émissions du pays d'origine et celles du pays d'adoption.

\section{DISTANCE CRITIQUE ET CONFORMISME CONTEXTUEL}

Interrogés sur leurs consommations quotidiennes, les jeunes issus de familles immigrées semblent se placer dans une ambivalence, entre une forme de réflexivité et de distance critique par rapport au consumérisme, d'une part, et le besoin d'être reconnus comme une partie intégrante du contexte dans lequel ils vivent tous les jours et qui comprend aussi ces mêmes comportements de consommation, d'autre part. Il s'agit d'une tension qui relève de l'attitude ambivalente plus générale entre différenciation et inclusion. Cette ambivalence, également mise en lumière dans d'autres contextes nationaux (Baumann, 1996), caractérise la vie quotidienne des jeunes avec des parents étrangers. L'exigence de se distinguer, injonction caractéristique d'une société très individualisée, se traduit ici par la tentative d'utiliser la différence culturelle inscrite dans sa propre biographie comme une ressource de distinction tout à fait compatible avec la participation et le sentiment d'inclusion. Cette attitude est bien visible dans les pratiques de consommation, au travers lesquelles une différence - qui parfois peut être subie ou stigmatisée - peut être plus facilement utilisée comme source de fascination. 
" Je suis noire mais je n'ai jamais eu de problèmes à l'école ou ailleurs, au contraire j'ai beaucoup d'amis, je les invite souvent chez moi pour goûter la cuisine de mon pays... on mange avec les mains : ça les amuse et ils aiment bien ça, ils sont fascinés aussi par ma maison, par certains objets... ils me posent souvent des questions sur mon pays, mais parfois c'est ma mère qui répond, car je ne connais pas bien l'histoire de l'Érythrée, puisque je suis née ici et je n'ai jamais vu le pays de mes parents » (Y. 18 ans $(\mathrm{F})$ parents érythréens).

Cette tentative d'être à la fois « égaux et différents » (Touraine, 1997) semble donc plus simple dans les pratiques de consommation plutôt que dans les définitions abstraites de sa propre identité. D'un côté les enquêtes quantitatives nationales montrent que les pratiques de consommation de ces jeunes sont tout à fait proches de celles des jeunes Italiens du même âge, surtout dans le secteur technologique - ordinateur, Internet, portable - ou dans l'utilisation de certaines marques très à la mode. Cependant les enquêtes qualitatives, comme la nôtre, montrent que l'utilisation d'Internet, du portable ou de certaines marques de vêtements peut avoir des significations partiellement différentes chez les jeunes descendants d'immigrés. D'ordinaire, la technologie est utilisée pour garder les contacts avec les amis et les proches dans le pays d'origine - e-mails et réseaux sociaux sont les moyens les plus utilisés - la possibilité de télécharger de la musique et des films gratuitement est une occasion pour garder un contact avec les atmosphères et l'imaginaire de la culture des parents, tout en ayant la possibilité d'explorer facilement et presque gratuitement les soundscapes et mediascapes d'autres cultures et pays. Par exemple beaucoup des jeunes que nous avons questionnés sont attirés par la culture japonaise, en particulier par les bandes dessinées et les mangas et rêvent de visiter le Japon. Des marques comme Nike ou Adidas sont, en plus du vêtement, également associées à des symboles, coiffures, accessoires qui rappellent leurs origines familiales ou leur attraction par d'autres sous-cultures (rasta et hip-hop notamment), donnant lieu à des compositions tout à fait originales et personnelles.

"J'ai une personnalité forte, je suis très indépendante, je ne suis pas médiocre,

j'aime certains livres, certaines choses, certains styles d'habillement un peu originaux, différents des autres, un style à moi [...]. Avec mon père le rapport est mauvais, mais sur l'habillement, il me laisse faire, il a confiance en moi, s'il me voit avec un habillement sombre-étrange, avec des tops et tout ça, il ne dit rien... » (I. 18 ans (F) parents bulgares).

Le désir de distinction peut se manifester aussi par une attitude radicalement critique envers les habitudes consuméristes des copains, le fait de pouvoir choisir autrement est donc interprété comme un signe de maturité personnelle et d'émancipation. Cette attitude semble plus fréquente chez les étudiants-salariés, c'est-à-dire chez les jeunes qui ont été précocement habitués par leurs parents à gagner leur argent de poche grâce à des petits boulots. Même s'il s'agit souvent de jeunes qui vivent dans des familles avec un pouvoir d'achat très limité, cette habitude d'être responsabilisé par rapport à l'argent ne semble entraîner ni ressentiment ni frustration. Au contraire, et une fois encore, cela semble être un signe de valorisation personnelle par rapport à un style de consommation perçu comme conformiste, imitatif et immature. 
" J'ai été habituée à travailler et étudier en même temps depuis que j'avais quatorze ans, je contribue au ménage de ma mère avec 200 euros par mois et j'en suis très fière... Mais je regarde mes copains (italiens) ici et je vois qu'ils ne sont jamais contents, pour eux ce n'est que la marque qui compte, ils regardent tout de suite ça, ou si tu as une belle voiture, sinon tu ne comptes rien... Je n'aime pas ça, alors je dis à mes copines à l'école: "mais bougez-vous! Travaillez! Il faut partir par le bas"... Je m'en fiche des pantalons Armani, c'est du gâchis, il y a de très beaux pantalons au marché dix fois moins chers » (L. 17 ans (F) parents domini-

cains).

"Mes parents savent que je suis un garçon responsable, si je vais dans un magasin avec mes amis et je vois quelque chose qui me plaît, je regarde d'abord le prix ; gaspiller cent euros pour un pantalon c'est une absurdité, à la limite il faut attendre les soldes [...]. Depuis une année je travaille deux fois par semaine dans un bureau près de chez moi, je nettoie le matin avant d'aller à l'école, je gagne 180 euros par mois et je suis indépendant par rapport à mes propres achats » (D. 18 ans (M) parents philippins).

La capacité de maintenir une distance critique par rapport à une culture de consommation hégémonique présuppose un certain degré d'autonomie individuelle qui est certainement favorisé par l'éducation familiale et son rapport à la consommation et à l'argent, mais aussi par un contexte qui ne pousse pas vers des identifications rigides. C'est dans ce cas en effet que la différence culturelle peut être perçue comme une ressource de distinction et de réflexivité sans être nécessairement le résultat d'un renversement du stigmate. Les situations qui se présentent plus fréquemment dans la vie de tous les jours semblent par contre caractérisées par une certaine ambiguïé entre une culture familiale centrée sur la responsabilisation dans l'utilisation de l'argent - justifiée par les sacrifices de l'histoire migratoire des parents - et un contexte quotidien fréquenté par leurs enfants notamment à l'école - qui les pousse vers des formes de conformisme contextuel, c'est-àdire temporaire et situé, qui s'exprime surtout dans l'habillement ou dans les accessoires technologiques jugés indispensables, comme le téléphone portable.

Par ailleurs filles et garçons présentent des dynamiques partiellement différentes par rapport aux choix des marques vestimentaires. Notamment les garçons ont une plus fréquente sociabilité de groupe. Les groupes masculins sont souvent caractérisés par une sous-culture qui s'exprime avec des codes vestimentaires plus contraignants, liés à l'expression du respect et de la virilité. Les règles de l'habillement présentent donc un caractère impératif et normatif, lié à l'appartenance et à la reconnaissance réciproque. La vie dans le groupe du même âge est au contraire moins exigeante pour les filles qui ont plus souvent des relations dyadiques; la tendance à changer souvent de vêtements rend aussi moins contraignante la référence à la marque et favorise le pluralisme des références culturelles et la personnalisation de la présentation de soi.

Cette différence de genre ne semble pas présente dans d'autres formes de consommation - musicales et technologiques notamment - cependant même la plus évidente normativité des codes d'habillement chez les garçons n'empêche pas des formes de réflexivité qui peuvent se manifester à l'extérieur du groupe : 
"Quand je suis avec mes amis, ou même à l'école, je suis toujours habillé d'une certaine façon, pantalon XL qui tombe, chaussures Nike ou Adidas énormes, blouson $X L$ toujours ouvert, lunettes de soleil... Je sors comme ça seulement en compagnie de mes copains, car dans la rue on te regarde mal... maintenant je suis habillé comme ça [normal], car je suis seul [...]. Les pantalons bas ce n'est pas pratique du tout, donc je ne les porte pas quand je suis seul » (P. 17 ans (M) parents domini-

cains).

De l'ensemble de ces narrations sur la consommation émerge une attitude pour laquelle l'aspiration à une qualité de vie représentée par le modèle « euro-américain » ne se renoue pas nécessairement à une assimilation passive, ou à un modèle de consommation dominé par l'omnipuissance des messages publicitaires. Parallèlement, revendiquer un espace d'expression de la différence culturelle et une distance critique par rapport au consumérisme ne signifie pas refuser le processus d'intégration dans la société italienne. La culture d'origine des parents et les références culturelles de la société italienne sont plutôt considérées comme des modèles pluriels à l'intérieur desquels il est possible de faire des choix, selon les circonstances et la situation présente.

Certes ces circonstances sont liées d'abord aux ressources économiques, sociales et culturelles familiales, ainsi qu'au contexte fréquenté quotidiennement par ces jeunes, à l'école et dans le quartier. Une analyse plus globale des pratiques et des cultures de la consommation ne peut donc négliger un regard sur les positions structurelles qu'influencent le choix et les significations de la culture matérielle.

\section{« DESCENDANTS D'IMMIGRÉS N'EST QU'UN MOT » : POSITIONS STRUCTURELLES ET INTERPRÉTATIONS SUBJEC- TIVES DE LA CONSOMMATION}

Dans un essai polémique qui commentait les études sur la jeunesse, Bourdieu (1980) affirmait que « la jeunesse n'est qu'un mot», pour remarquer la nécessité de ne pas oublier les différences de classe et de statut à l'intérieur d'une classe d'âge qui, dans les années 1970, présentait des sous-cultures - ouvrières ou d'élite - sans doute plus marquées qu'aujourd'hui. En effet, dans la perspective des études sur la consommation réalisées par Bourdieu (1979), la classe, donc la position économique occupée dans la structure sociale, constituait une sorte de variable indépendante par rapport à la reproduction du statut social, donc aux goûts et aux esthétiques qui caractérisent aussi la consommation. À l'opposé de cette position se situent des interprétations plus confiantes envers les capacités subjectives de libre choix individuel parmi un ensemble de références culturelles et esthétiques. Ces dernières sont mises à la disposition de tous par la société globalisée et postmoderne (Featherstone, 1990), selon une perspective qui, contrairement à l'approche de Bourdieu, tend à sous-évaluer l'influence de la classe sociale et des positions occupées par les acteurs dans les structures de la société.

Du coup, les recherches disponibles sur la consommation des immigrés et de leurs enfants tendent aussi à se placer à l'intérieur de l'ambivalence entre le déterminisme de la position structurelle occupée (revenu de la famille, quartier de résidence, niveau 
d'instruction) et les interprétations subjectives et relativement autonomes de la culture matérielle. Cette ambivalence reste donc au centre de toutes les études sur la consommation, mais justement pour cette raison les pratiques de consommation sont un point de vue privilégié pour étudier à la fois les aspects interprétatifs et structuraux, c'est-à-dire les marges d'autonomie et les formes de domination qui caractérisent le rapport de l'acteur à la culture matérielle.

De son côté l'étude de la consommation des familles immigrées peut aider à mettre à jour les approches sur la reproduction des habitudes de consommation. En effet, la perspective de la différence culturelle peut mieux éclairer comment dans une société potentiellement pluraliste et multiculturelle l'élaboration des goûts et les interprétations des significations de la consommation produisent des dissonances. Par conséquent, des positions de classe ou des identifications culturelles rigides et autoréférentielles ne sont pas forcément et inévitablement reproduites dans la consommation (Lahire, 2004). Cela semble encore plus vraisemblable pour les jeunes descendants d'immigrés dans un contexte comme celui de l'Italie qui reste pour l'instant suffisamment ouvert aux formes de multiculturalisme quotidien.

Certes la position socio-économique de la famille et le capital culturel et social dont elle dispose - y compris en termes de réseaux ethniques d'aide mutuelle - restent importants pour déterminer les ressources et les motivations à disposition des enfants : en particulier, les conseils sur les filières d'études les plus favorables à la mobilité sociale et le contrôle sur les résultats scolaires, mais aussi l'ouverture d'esprit nécessaire pour garantir la capacité de choisir entre identifications culturelles plurielles sans s'enfermer dans une identité ethnique défensive, ou une identification passive et mimétique de la culture occidentale.

\begin{abstract}
"Mes parents ont beaucoup insisté sur mon éducation, ils me voient déjà avocat ou médecin, donc il faut que je travaille beaucoup à l'école pour ne pas les décevoir ; c'est un peu lourd tout ça, mais c'est juste... En même temps je dois faire attention avec l'argent, car maintenant je gagne moi-même mon argent de poche avec des petits boulots, par exemple une fois par semaine, le mercredi, je vide la poubelle dans un restaurant très tôt le matin, mais c'est bien payé, c'est mon oncle qui m'a aidé » (J. 18 ans (M) parents philippins).
\end{abstract}

La plupart des familles immigrées, desquelles sont issus les jeunes que nous avons interviewés, présentent des formes d'intégration subalterne dans la société italienne (Ambrosini, 2001). Les métiers des parents de ces jeunes sont souvent peu qualifiés et parfois précaires. Beaucoup de ces migrants, bien que diplômés dans leur pays, ont accepté des professions à bas contenu de connaissance, comme celle d'ouvriers, de petits commerçants, ou bien ils travaillent dans les services d'aide à la personne. Ceci a parfois signifié une chute de statuts par rapport au pays d'origine, mais cela a établi aussi une culture de la dignité personnelle et du travail qui a été transmise aux enfants et cela a évité chez eux des sentiments d'infériorité ou de frustration. Cette revendication de dignité s'exprime aussi par une approche réflexive et responsable envers la consommation et par une responsabilisation précoce dans l'usage de l'argent. Dans d'autres cas au contraire, l'installation dans la société italienne a signifié pour ces parents l'accès à une culture de la consommation considérée comme moderne et opposée aux traditions du pays : cette attitude, plus favorable aux pratiques consuméristes, a inévitablement influencé leurs enfants. 
À l'intérieur des familles immigrées, nous sommes donc face à une socialisation à la consommation diversifiée selon les biographies et les histoires migratoires, dans laquelle les dissonances entre position économique et capital socioculturel sont fréquentes. Pour leurs enfants cependant, le fait de vivre dans une époque historique dans laquelle les références culturelles sont bien davantage plurielles et transnationales, transforme les styles de consommation dans un champ où les comportements pluralistes et omnivores deviennent le vrai signe d'intégration. Au contraire, des formes d'imitation ou de refus manifestent l'enfermement dans un modèle pauvre d'imagination, monolithique et uniforme (Hannerz, 1992).

" Je pense qu'avoir des parents étrangers c'est une chance; le fait de pouvoir circuler entre plusieurs cultures est une richesse ; ma mère est croate, mon père est serbe, j'ai un grand-père italien, je suis né dans un pays et je vis dans un autre, j'ai des amis de nationalité différente : cela enrichit beaucoup ma vie [...]. À la maison on mélange la nourriture, les programmes de la télé, je suis curieux, ça ne m'intéresse pas d'être enfermé dans un modèle, dans une histoire... » (J. 18 ans (M)

parents serbo-croates).

"Je pense que si tu n'as pas suffisamment d'argent pour acheter une chose tu dois renoncer, je ne suis pas d'accord avec mes voisins qui achètent tout avec des emprunts et qui sont pleins de dettes... je n'aime pas cette culture de la mode, où tout le monde doit avoir les mêmes choses, ça me semble débile... J'essaie de choisir selon ma personnalité, mon goût, je sélectionne... Je porte le voile, car c'est mon choix, mais je ne renonce pas à soigner mon aspect ou à acheter un sac élégant si je peux... ton style doit refléter ta personnalité » (S. 18 ans $(\mathrm{F})$ mère polonaise, père

égyptien).

Notre étude révèle ainsi un équilibre complexe et instable entre, d'un côté, les positions économiques de la famille et les interprétations subjectives de la consommation et, d'un autre coté, la référence aux imaginaires commerciaux occidentaux et la référence à la culture d'origine des parents comme forme de distinction. L'interprétation que chacun de ces jeunes descendants d'immigrés, nés ou arrivés en Italie, donne à ses appartenances et fidélités multiples, ainsi que l'image de soi et de sa propre différence que chaque famille projette à l'extérieur et reçoit en retour, font également partie de cet équilibre changeant.

Il en résulte alors un ensemble de pratiques et de cultures diversifiées de la consommation chez les enfants d'immigrés qui ne se réduisent pas du tout à un choix entre l'acculturation-imitation et le refus-enfermement communautaire. Ce résultat consiste plutôt en une gamme d'interprétations du rapport à la culture matérielle et à la différence culturelle. Dans ce sens la biographie d'enfant d'immigrés peut toujours jouer comme une ressource réflexive et la culture marchande peut toujours se transformer dans un espace de micro-tactiques d'innovation et de résistance, selon la perspective déjà décrite par De Certeau (1978). 


\section{CONCLUSION}

La recherche sur la consommation des enfants d'immigrés présentée ici montre une situation qui semble plus caractérisée par la génération à laquelle ces jeunes appartiennent qu'à leurs origines sociales ou familiales. L'utilisation quotidienne d'Internet, de l'ordinateur, de l'iPod ou du téléphone portable, des goûts esthétiques et musicaux diversifiés et pluriels, montrent des pratiques de consommation tout à fait semblables à celles des jeunes italiens « de souche ». Ce sont des pratiques qui vont au-delà de l'imitation d'un modèle hégémonique et caractérisé par la subordination à une culture publicitaire de la marque. Cette culture semble au contraire plus répandue chez les jeunes italiens plutôt que chez les enfants d'immigrés, qui peuvent toujours se détacher réflexivement des attitudes les plus consuméristes en s'appuyant sur l'originalité et la différence de leurs biographies familiales.

Dans ce travail exploratoire, nous avons essayé de cueillir surtout les aspects interprétatifs des pratiques de consommation de cette génération de « nouveaux Italiens » : le résultat est un panorama complexe où à des pratiques apparemment conformes s'ajoutent des interprétations, voire des usages plus détachés et critiques des biens de consommation. Certes il s'agit d'un équilibre fragile, qui risque à tout moment de basculer vers des formes d'enfermement identitaire (différentialiste ou imitatif) chaque fois que la situation oblige à prendre une position plus nette. En effet la différence culturelle dont ces jeunes sont porteurs doit toujours se rapporter à une culture de la consommation majoritaire et d'origine occidentale (Perry, 2001).

Cependant, la situation italienne contemporaine - localement diversifiée et culturellement hétérogène, ainsi que moins contaminée par un imaginaire colonial - semble pour l'instant favoriser des situations de multiculturalisme quotidien qui se manifestent aussi dans la relation à la culture matérielle. Cette situation et cette interprétation davantage pluraliste de la consommation que nous avons pu enregistrer dans le contexte milanais ne sont peut-être pas automatiquement extensibles à d'autres contextes locaux italiens - bien que confortées par les résultats d'autres études ethnographiques réalisées en Italie sur la vie quotidienne des descendants d'immigrés - et elle est sans doute présente également dans d'autres contextes nationaux européens. Les processus d'intégration et d'acculturation à la consommation n'apparaissent donc pas comme des parcours déjà établis, par rapport auxquels on peut se placer seulement selon une perspective alternative d'inclusion ou d'exclusion. Au contraire, les positions et les formes de déterminations sociales interagissent avec les interprétations subjectives et les formes de réflexivité et de prise de distance qui se fondent sur les pratiques-mêmes de consommation. Dans ce sens, être enfant d'immigrés et être capable de circuler entre plusieurs codes culturels introduit des dissonances et des éléments de contraste qui facilitent l'autonomie, chaque fois que cette possibilité se trouve favorisée par un contexte, familial et public, ouvert et réceptif. Notre recherche montre donc que, bien que ces enfants d'immigrés grandissent, dans la plupart de cas, dans des familles se trouvant dans une position sociale subalterne et avec un pouvoir d'achat limité, la consommation reste un terrain possible d'une participation sociale qui ne veut pas être subalterne par rapport à un modèle imposé, ni défensive et enfermée dans la référence à des marqueurs ethniques. 


\section{Références bibliographiques}

AMBROSINI Maurizio (2001) La fatica di integrarsi, Bologna, Il Mulino, 216 p.

AMBROSINI Maurizio e MOLINA Stefano (Dir.) (2004) Seconde generazioni, Torino, Edizioni Giovanni Agnelli, 185 p.

APPADURAI Arjun (1996) Modernity at Large: Cultural Dimensions of Globalization, MinneapolisLondon, Univ. of Minnesota Press, 248 p.

BATTEGAY Alain (2003) Les recompositions d'une centralité commerçante immigrée : la Place du Pont à Lyon, Revue Européenne des Migrations Internationales, 9 (2), pp. 9-22.

BAUMANN Gerd (1996) Contesting Culture. Discourses of Identity in Multi-Ethnic London, Cambridge, Cambridge Univ. Press, 215 p.

BESOZZI Elena, COLOMBO Maddalena e SANTAGATI Maria Grazia (2009) Giovani stranieri, nuovi cittadini. Le strategie di una generazione, Milano, Francoangeli, 256 p.

BOSISIO Roberta, COLOMBO Enzo, LEONINI Luisa e REBUGHINI Paola (2005) Stranieri \& Italiani, Roma, Donzelli, $199 \mathrm{p}$.

BOURDIEU Pierre (1980) Questions de Sociologie, Paris, Minuit, 277 p.

BOURDIEU Pierre (1979) La distinction. Critique sociale du jugement, Paris, Minuit, 670 p.

BREWER John and TRENTMANN Frank (Eds.) (2006) Consuming Cultures, Global Perspectives: Historical Trajectories, Transnational Exchanges, Oxford and New York, Berg, 317 p.

CANCLINI Nestor G. (1998) Culture ibride:strategie per entrare e uscire dalla modernità, Milano, Guerini, 294 p.

CARITAS MIGRANTES (2009) XIX Rapporto sull'immigrazione, Dossier statistico 2009, Milano, Edizioni Idos, $512 \mathrm{p}$.

CHIN Elizabeth (2001) Purchasing Power. Black Kids and American Consumer Culture, Minneapolis, Univ. of Minnesota Press, $280 \mathrm{p}$.

COLOMBO Enzo, DOMANESCHI Lorenzo e MARCHETTI Chiara (2009) Una nuova generazione di italiani. L'idea di cittadinanza tra i giovani figli di immigrati, Milano, FrancoAngeli, $144 \mathrm{p}$.

COLOMBO Enzo, LEONINI Luisa and REBUGHINI Paola (2009) Different But Not Stranger: Everyday Collective Identification among Adolescent Children of Immigrants in Italy, Journal of Ethnic and Migration Studies, 35 (1), pp. 37-59.

DAVILA Arlene (2001) Latinos Inc. The Marketing and Making of a People, Berkeley, Univ. of California Press, 308 p.

DE CERTEAU Michel (1978) L'invention du quotidien, Paris, Gallimard (2 vol.), $374 \mathrm{p}$ et $415 \mathrm{p}$.

FEATHERSTONE Mike (1990) Lifestyles and Consumer Culture, Sociology, 24 (1), pp. 5-22.

HANNERZ Ulf (1992) Cultural Complexity: Studies in the Sociological Organization of Meaning, New York, Columbia University Press, 347 p.

LAHIRE Bernard (2004) La culture des individus. Dissonances culturelles et distinction de soi, Paris, La Découverte, 777 p.

LANGLOIS Simon (2002) Nouvelles orientations en sociologie de la consommation, L'année sociologique, 52, pp. 83-103.

LAPEYRONNIE Didier (1998) L'ordre de l'informe. La construction sociale et politique du racisme dans la société française, Hommes et Migrations, 1211, pp. 68-82.

LAPEYRONNIE Didier (2009) Ghetto urbain. Ségrégation, violence, pauvreté en France aujourd'hui, Paris, Laffont, $624 \mathrm{p}$.

LEONINI Luisa e REBUGHINI Paola (2010) Legami di nuova generazione. Relazioni famigliari e pratiche di consumo tra i giovani discendenti di migranti, Bologna, Il Mulino, $240 \mathrm{p}$.

MARTUCCELLI Danilo (2006) Forgé par l'épreuve, Paris, Armand Colin, 478 p.

MILLER Daniel (Ed.) (1995) Acknowledge Consumption: a Review of New Studies, Oxford, Blackwell, 341 p. 
PAYET Jean-Paul (2008) Immigration et école, in Dictionnaire de l'éducation, Paris, Presses Universitaires de France, pp. 367-371.

PERRY Pamela (2001) White means never having to say you're ethnic: White youth and the construction of "cultureless" identities, Journal of Contemporary Ethnography, 30 (1), pp. 56-91.

PORTES Alejandro, FERANDEZ-KELLY Patricia and HALLER William (2009) The adaptation of the immigrant second generation in America: a theoretical overview and recent evidence, Journal of Ethnic and Migration Studies, 35 (7), pp. 1077-1104.

PORTES Alejandro, FERANDEZ-KELLY Patricia and HALLER William (2005) Segmented assimilation on the ground: the new second generation in early adulthood, Ethnic and Racial Studies, 28 (6), pp. 1000-1040.

PORTES Alejandro and RUMBAUT Ruben (2001) Legacies. The Story of the Immigrant Second Generation, Berkeley, University of California Press, 353 p.

QUEIROLO PALMAS Luca (2006) Prove di seconde generazioni. Giovani di origine immigrata tra scuole e spazi urbani, Milano, Francoangeli, 208 p.

RAULIN Anne (2000) L'ethnique est quotidien. Diasporas, marchés et cultures métropolitaines, Paris, L'Harmattan, 229 p.

REBUGHINI Paola (2010) Identificarsi attraverso gli oggetti. Cultura materiale e pratiche di consumo tra i discendenti dei migranti, in Luisa Leonini e Paola Rebughini Eds., Legami di nuova generazione, Bologna, Il Mulino, pp. 127-168.

ROULLEAU-BERGER Laurence (2009) Le seconde generazioni di fronte alla precarizzazione de all'etnicizzazione del lavoro in Francia, Sociologia e Politiche Sociali, 12 (1), pp. 47-57.

TARRIUS Alain (2002) La mondialisation par le bas, Paris, Balland, 220 p.

TOURAINE Alain (1997) Pourrons-nous vivre ensemble? Paris, Fayard, 395 p.

VALTOLINA Giovanni e MARAZZI Antonio (2006) Appartenenze multiple. L'esperienza dell'immigrazione nelle nuove generazioni, Milano, FrancoAngeli, $240 \mathrm{p}$.

WARDE Alan (2005) Consumption and the theory of practice, Journal of Consumer Culture, 5, pp. 131-54.

WIEVIORKA Michel (2008) L'intégration : un concept en difficulté, Cahiers Internationaux de Sociologie, 75, pp. 221-40.

ZUKIN Sharon (2004) Point of Purchase: How Shopping Changed American Culture, New York, Routledge, $336 \mathrm{p}$.

ZUKIN Sharon and SMITH-MAGUIRE Jennifer S. (2004) Consumer and Consumption, Annual Review of Sociology, 30, pp. 173-947. 


\title{
Consommation et cultures de la différence chez les jeunes descendants d'immigrés. Réflexions à partir du cas italien
}

\author{
Paola Rebughini
}

\begin{abstract}
Cet article s'occupe des pratiques et cultures de la consommation chez les jeunes descendants d'immigrés en Italie. En effet les modèles de la consommation représentent une perspective particulièrement intéressante pour observer à la fois les aspects structuraux de l'intégration, liés à la position socioéconomique des familles immigrées, et les aspects interprétatifs et symboliques de la différence culturelle, aspects qui caractérisent les pratiques quotidiennes des jeunes avec des parents étrangers. L'article se base sur une recherche spécifiquement dédiée aux pratiques et aux cultures de la consommation chez cette catégorie de jeunes dans la région milanaise et plus généralement sur les résultats de cinq années (2004-2009) de recherche sur cette génération de jeunes en Italie.
\end{abstract}

\section{Consumption and Cultures of Difference among the Children of Immigrants. Reflections from the Italian Case}

\author{
Paola Rebughini
}

The aim of this article is to present the cultures and practices of consumption among young people with immigrant origins in Italy. Models of consumption can be an interesting way to study at the same time subjective and contingent aspects of consumption practices and structural aspects of consumption behaviour, linked to economic, social and cultural capital of families. This article is based on a specific research about consumption practices of the children of immigrants in the urban area of Milan and more generally on the results of five years of research (2004-2009) about the second generation in Italy.

\section{Comsumos y culturas de la diferencia de los hijos de inmigrantes. Reflexión sobra lo caso Italiano}

\section{Paola Rebughini}

Esta investigación se concentra sobre las prácticas y las culturas del consumo de los jóvenes descedientes de inmigrantes en Italia. De todo modo, los modelos de consumo representen una perspectiva interesante para observar en el mismo tiempo los aspectos estructurales de la integración - ligadas a la posición socio-económica de las familias - y los aspectos mas interpretativos y simbólico de la diferencia cultural, que caraterizan las prácticas cotidianas de los jóvenes con padres estranjeros. La investigación sobre los consumos ha sido desarollada con los resultados de una investigación de la duración de cinco años (2004-2009) sobre la generación de los hijos de inmigrantes en Italia y sobre una investigación aprofondida del consumo en la región de Milan. 